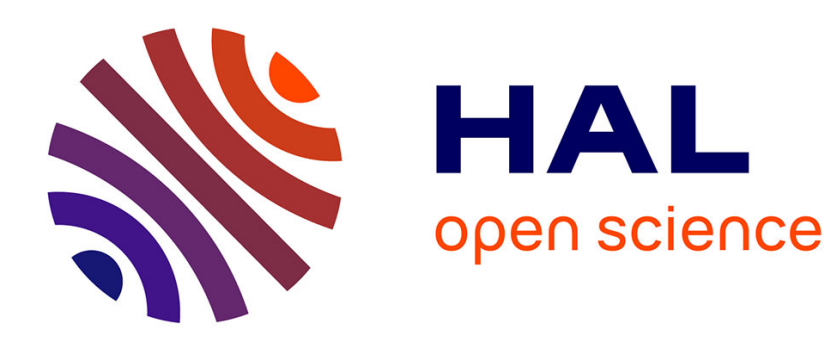

\title{
Optical Transverse Injection in Laser-Plasma Acceleration
}

R. Lehe, Agustin Lifschitz, X. Davoine, Cédric Thaury, Victor Malka

\section{To cite this version:}

R. Lehe, Agustin Lifschitz, X. Davoine, Cédric Thaury, Victor Malka. Optical Transverse Injection in Laser-Plasma Acceleration. Physical Review Letters, 2013, 111 (8), pp.085005. 10.1103/PhysRevLett.111.085005 . hal-01163765

\section{HAL Id: hal-01163765 \\ https://hal.science/hal-01163765}

Submitted on 17 Jul 2015

HAL is a multi-disciplinary open access archive for the deposit and dissemination of scientific research documents, whether they are published or not. The documents may come from teaching and research institutions in France or abroad, or from public or private research centers.
L'archive ouverte pluridisciplinaire HAL, est destinée au dépôt et à la diffusion de documents scientifiques de niveau recherche, publiés ou non, émanant des établissements d'enseignement et de recherche français ou étrangers, des laboratoires publics ou privés. 


\title{
Optical Transverse Injection in Laser-Plasma Acceleration
}

\author{
R. Lehe, ${ }^{1, *}$ A. F. Lifschitz, ${ }^{1}$ X. Davoine, ${ }^{2}$ C. Thaury, ${ }^{1}$ and V. Malka ${ }^{1}$ \\ ${ }^{1}$ Laboratoire d'Optique Appliquée, ENSTA ParisTech-CNRS UMR7639-École Polytechnique, \\ 828 Boulevard des Maréchaux, Palaiseau 91762, France \\ ${ }^{2}$ Commissariat à l'énergie atomique, DAM, DIF, Arpajon F-91297, France
}

(Received 20 March 2013; published 23 August 2013)

\begin{abstract}
Laser-wakefield acceleration constitutes a promising technology for future electron accelerators. A crucial step in such an accelerator is the injection of electrons into the wakefield, which will largely determine the properties of the extracted beam. We present here a new paradigm of colliding-pulse injection, which allows us to generate high-quality electron bunches having both a very low emittance $(0.17 \mathrm{~mm} \cdot \mathrm{mrad})$ and a low energy spread $(2 \%)$, while retaining a high charge $(\sim 100 \mathrm{pC})$ and a short duration ( $3 \mathrm{fs}$ ). In this paradigm, the pulse collision provokes a transient expansion of the accelerating bubble, which then leads to transverse electron injection. This mechanism contrasts with previously observed optical injection mechanisms, which were essentially longitudinal. We also specify the range of parameters in which this new type of injection occurs and show that it is within reach of existing highintensity laser facilities.
\end{abstract}

DOI: 10.1103/PhysRevLett.111.085005

PACS numbers: 52.38.Kd, 41.75.-i, 52.65.Rr

In view of past results [1-4], laser-wakefield acceleration appears as a compact alternative to conventional accelerators. Yet, a key requirement for laser-wakefield accelerators and their applications [5] is to improve the stability and quality (total charge, energy dispersion, and emittance) of the beam. This is particularly true for the prospective use of laser-wakefield electrons in freeelectron lasers [6], which simultaneously requires a high amount of charge, a low energy spread, and a low emittance [7]. These properties depend to a large extent on how electrons are injected into the accelerating bubble. However, no known injection technique can provide high charge, low energy dispersion, low emittance, and high stability at the same time.

For instance, transverse self-injection-in which the nonlinear propagation of the laser pulse is responsible for the injection of off-axis electrons-leads to large injected charges (100-200 pC) [8,9] but with a large energy spread and strong shot-to-shot fluctuations. Alternatively, longitudinal self-injection [10] produces stable beams but with low charges $(\sim 5 \mathrm{pC})$. It was also recently reported [11] that self-injection can produce very low emittances $(0.1 \mathrm{~mm}$. $\mathrm{mrad})$, yet again with very low charge $(0.4 \mathrm{pC})$. This tradeoff between high charge and low emittance and energy spread also applies to controlled injection techniques, such as density-gradient injection [12,13], ionizationinduced injection [14], Trojan horse injection [15,16], or optical injection [17-20]. In optical injection, for example, the electrons are injected during the collision of the driving laser pulse with a counterpropagating pulse, making it a remarkably stable scheme and producing very low energy dispersions. Yet, in the regime of parameters that were probed up to now, the produced beams were characterized by a low amount of charge (a few tens of pC) $[20,21]$ and a relatively large emittance $(\sim 0.5 \mathrm{~mm} \cdot \mathrm{mrad})$ [21]. These limitations can, however, be overcome, and in this Letter, we report on a new regime of optical injection which meets both the requirements of low emittance $(0.17 \mathrm{~mm} \cdot \mathrm{mrad})$ and relatively high charge $(\sim 50-100 \mathrm{pC})$, while retaining the low energy dispersion (2\%) associated with collidingpulse schemes.

This particular regime is also characterized by a radically different mechanism of injection. In previous regimes of colliding-pulse schemes (both warm injection [22-24] and cold injection [25-27]), the dynamics of injection is essentially longitudinal; i.e., only the longitudinal momentum and position of the electrons determine its injection. If, thanks to the collision, an electron can cross the separatrix associated with the wakefield, then it is trapped, but its transverse behavior has no direct influence on the injection process. Moreover, in these regimes, the pulse collision strongly affects that small fraction of electrons which is then injected, but the background electrons (those forming the bubble and its sheath) are usually much less affectedand if they are, this tends to degrade injection rather than improve it [28].

On the contrary, we show here that in the abovementioned new regime, the bubble as a whole is strongly affected and that this deformation is essential for injection. As shown below, the pulse collision reduces the efficiency of the transverse ponderomotive force. As a result, the whole bubble becomes smaller. Once the pulse collision is over, the ponderomotive force retrieves its initial efficiency and the bubble recovers its original shape; i.e., it expands back. The expansion of the bubble then triggers transverse injection. This injection is thus similar to the one described in evolving-bubble theories [29-32], which demonstrated that an expanding bubble indeed tends to 
trigger transverse injection. In fact, previous work already proposed to control transverse injection by controlling the expansion of the bubble, e.g., using a density down-ramp [12] or an external magnetic field [33,34]. Yet, this Letter shows that a pulse collision can also be used to trigger a transient and fast deformation of the bubble, and thus to control transverse injection. (We will refer to this mechanism as optical transverse injection in the rest of this Letter.)

In order to illustrate optical transverse injection, we ran particle-in-cell simulations using the quasicylindrical code CALDER CIRC [35]. Importantly, we use here a modified Maxwell solver (i.e., distinct from the standard Yee scheme), so as to avoid spurious growth of emittance caused by numerical artifacts [36]. In the situation we consider, the driving laser pulse $\left(\lambda_{0}=0.8 \mu \mathrm{m}\right)$ is focused to a waist $w_{0}=7.8 \mu \mathrm{m}$ into a plasma of density $n=$ $10^{-3} n_{c}=1.75 \times 10^{18} \mathrm{~cm}^{-3}$. Its FWHM duration is $\tau_{0}=$ $30 \mathrm{fs}$ and its total energy is $1.0 \mathrm{~J}$, corresponding to $a_{0}=4$ at its peak, and consequently leading to the formation of a clear-cut plasma bubble in its wake. Close to its focal plane, this pulse collides with a counterpropagating pulse having similar properties $\left(\tau_{1}=\tau_{0}, w_{1}=w_{0}, \lambda_{1}=\lambda_{0}\right)$ but much less intensity $\left(a_{1}=0.2\right)$. Here, both pulses are circularly polarized-with opposite senses of rotation, so that a standing beat wave is created when they collide. (Similar results were observed for parallel linear polarizations, which also produce a standing beat wave.)

Four consecutive snapshots of this simulation are shown in Fig. 1, along with a plot of the transverse and longitudinal size of the bubble versus time. The bubble distinctively shrinks and reexpands, first radially (between $t=40 \mathrm{fs}$ and $t=80 \mathrm{fs}$ ) and then longitudinally (between $t=100 \mathrm{fs}$ and $t=150 \mathrm{fs}$ ). This traveling deformation is caused by the same group of electrons that were inside the laser pulse at $t=0$ (thus feeling the pulse collision, and therefore a weaker ponderomotive force, as explained later) and then travel along the sheath towards the back of the bubble. At $t=150 \mathrm{fs}$, the bubble expands longitudinally, and that lowers the threshold for injection. Consistently, although some injected electrons can be already observed at $t=$ $99 \mathrm{fs}$, the majority of the injection occurs at $t=150 \mathrm{fs}$. A close inspection of the trajectories of the corresponding particles reveals that this second injection at $t=$ $150 \mathrm{fs}$ is transverse, i.e., that the injected electrons originate from the off-axis position and travel around the bubble before being injected, which is in accordance with evolving-bubble models [29-32].

As mentioned above, the observed shrinkage of the bubble is due to the reduced efficiency of the ponderomotive force during the collision. This reduced efficiency can be explained through the equations of motion of the electrons in the laser pulse [decomposed here over the radial $(r)$ and longitudinal $(z)$ directions]:
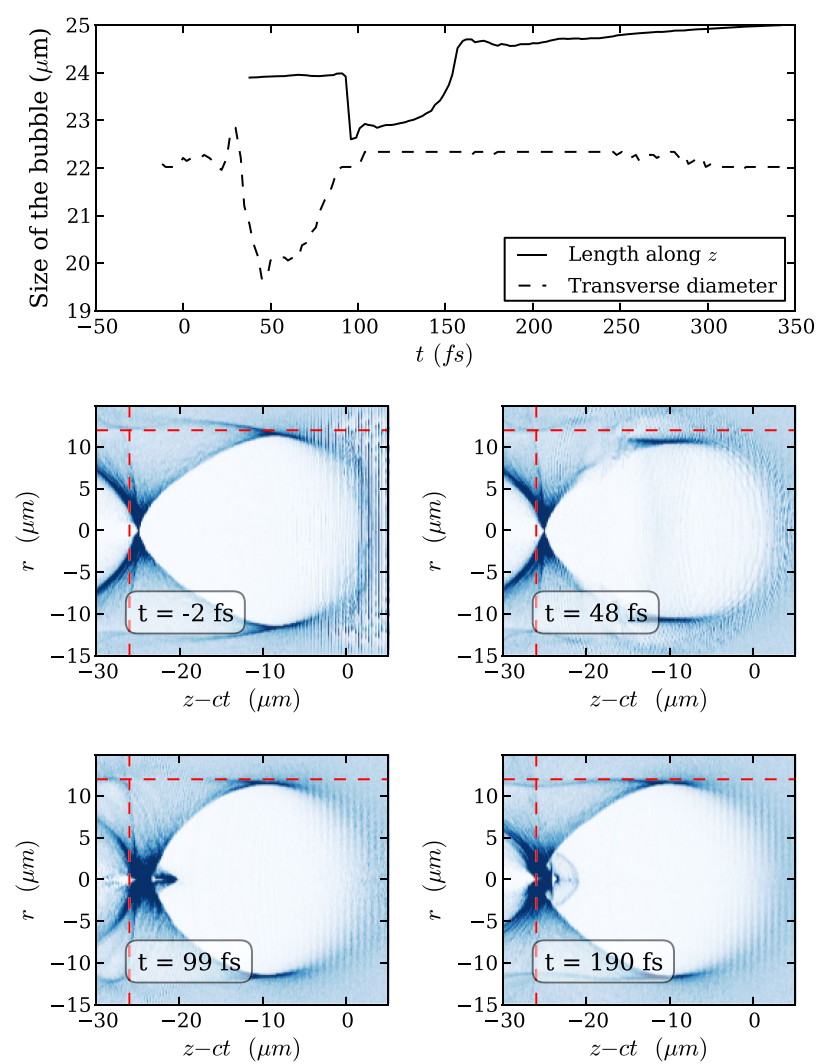

FIG. 1 (color online). Top panel: Evolution of the size of the bubble in the transverse direction (dashed line) and longitudinal direction (solid line) versus time. (The pulse collision occurs at $t=0$.) Bottom panels: Snapshots of the electron density at different times. The dashed red lines are visual supports that help evaluate the deformation of the bubble.

$$
\begin{array}{ll}
\frac{d p_{z}}{d t}=-\frac{1}{\gamma} \frac{\partial \vec{a}^{2}}{\partial z}, & \frac{d z}{d t}=\frac{p_{z}}{\gamma}, \\
\frac{d p_{r}}{d t}=-\frac{1}{\gamma} \frac{\partial \vec{a}^{2}}{\partial r}, & \frac{d r}{d t}=\frac{p_{r}}{\gamma},
\end{array}
$$

where $\vec{a}$ is the sum of the vector potentials of both pulses characterized by the same wave number $k_{0}$ and duration $\tau_{0}$ :

$$
\begin{aligned}
\vec{a}= & \frac{a_{0}}{\sqrt{2}}\left(\cos \zeta_{-} \vec{u}_{x}-\sin \zeta_{-} \vec{u}_{y}\right) e^{-\left((z-c t)^{2} /\left(c \tau_{0}^{\prime}\right)^{2}\right)-\left(r^{2} / w_{0}^{2}\right)} \\
& +\frac{a_{1}}{\sqrt{2}}\left(\cos \zeta_{+} \vec{u}_{x}+\sin \zeta_{+} \vec{u}_{y}\right) e^{-\left((z+c t)^{2} /\left(c \tau_{0}^{\prime}\right)^{2}\right)-\left(r^{2} / w_{1}^{2}\right)},
\end{aligned}
$$

where $\zeta_{-}=k_{0}(z-c t), \quad \zeta_{+}=k_{0}(z+c t), \quad$ and $\quad \tau_{0}^{\prime}=$ $\tau_{0} / \sqrt{2 \ln (2)}$. As a consequence,

$$
\begin{aligned}
\vec{a}^{2}= & \frac{a_{0}^{2}}{2} e^{-2\left((z-c t)^{2} /\left(c \tau_{0}^{\prime}\right)^{2}\right)-2\left(r^{2} / w_{0}^{2}\right)} \\
& +\frac{a_{1}^{2}}{2} e^{-2\left((z+c t)^{2} /\left(c \tau_{0}^{\prime}\right)^{2}\right)-2\left(r^{2} / w_{1}^{2}\right)} \\
& +\frac{a_{0} a_{1}}{2} \cos \left(2 k_{0} z\right) e^{-8\left(z c t /\left(c \tau_{0}^{\prime}\right)^{2}\right)-\left(r^{2} / w_{1}^{2}\right)-\left(r^{2} / w_{0}^{2}\right)} .
\end{aligned}
$$


The equations of motion can be simplified in two asymptotic cases. First, long before or long after the collision $\left(t \ll-\tau_{0}^{\prime}\right.$ or $t \gg \tau_{0}^{\prime}$ ), the colliding pulse is absent from the region of the driving pulse $\left[c\left(t-\tau_{0}^{\prime}\right)<z<c\left(t+\tau_{0}^{\prime}\right)\right]$, and the last two terms of Eq. (4) can be neglected. Therefore, in this region, the ponderomotive force only depends on $r$ and $\eta=t-z / c$, and as a result, $\gamma-p_{z}=1$ is a constant of the motion [37]. This, in turn, implies that $d \eta / d t=1 / \gamma$ and Eq. (2) can be written in the form

$$
\frac{d p_{r}}{d \eta}=\frac{2 r}{w_{0}^{2}} a_{0}^{2} e^{-2\left(\eta^{2} / \tau_{0}^{\prime 2}\right)-2\left(r^{2} / w_{0}^{2}\right)}, \quad \frac{d r}{d \eta}=p_{r} .
$$

Conversely, during the collision $\left(-\tau_{0}^{\prime}<t<\tau_{0}^{\prime}\right.$ and $-c \tau_{0}^{\prime}<z<c \tau_{0}^{\prime}$ ), the colliding pulse cannot be neglected anymore, and in fact the longitudinal ponderomotive force $\partial \vec{a}^{2} / \partial z$ is dominated by the third term of Eq. (4). [The contribution of the third term to $\partial \vec{a}^{2} / \partial z$ is of order $k_{0} a_{0} a_{1}$, whereas that of the first term is of order $2 a_{0}^{2}(z-$ $c t) /\left(c \tau_{0}^{\prime}\right)^{2}$.] This longitudinal ponderomotive force corresponds to that of the standing beat wave and holds the electrons in the minima of the interference pattern. Therefore, $d z / d t \simeq 0$ and $d \eta / d t \simeq 1$. On the other hand, the radial ponderomotive force $\partial \vec{a}^{2} / \partial r$ is still dominated by the first term of Eq. (4) as long as $a_{1} \ll a_{0}$, and Eq. (2) can be written as

$$
\frac{d p_{r}}{d \eta}=\frac{1}{\gamma} \frac{2 r}{w_{0}^{2}} a_{0}^{2} e^{-2\left(\eta^{2} / \tau_{0}^{\prime 2}\right)-2\left(r^{2} / w_{0}^{2}\right)}, \quad \frac{d r}{d \eta}=\frac{p_{r}}{\gamma} .
$$

Equations (5) (which applies before and after the pulse collision) and (6) (which applies during the collision) have to be integrated from the moment when an electron enters the pulse $\left(\eta<-\tau_{0}^{\prime}\right)$ to the moment when this electron exits the pulse $\left(\eta>\tau_{0}^{\prime}\right)$. Because of the factors $\gamma$ in Eq. (6), the electron will exit the pulse with a relatively lower transverse momentum $p_{r}$ and a lower radius $r$. This thus demonstrates that the pulse collision makes the transverse ponderomotive force less effective, as observed in Fig. 1.

Let us now turn to the properties of the bunches that are generated by optical transverse injection. Figure 2 displays the spectrum of the electrons obtained in the simulation considered here. Two types of electrons can be seen: a well-defined peak at $27 \mathrm{MeV}$, located at $z-c t=-25 \mu \mathrm{m}$ and corresponding to the transverse injection at $t=150 \mathrm{fs}$ from Fig. 1, and a long tail between 16 and $26 \mathrm{MeV}$, located around $z-c t=-23 \mu \mathrm{m}$ and corresponding to the injection at $t=99$ fs. Importantly, the total charge contained in the peak is $50 \mathrm{pC}$ while that in the tail is $13 \mathrm{pC}$, meaning that transverse injection largely dominates in these conditions.

The electrons obtained by optical transverse injection presents high-quality features: the rms duration of the bunch is as short as 3 fs (and is a consequence of the short duration of the collision) and its absolute rms energy spread is $0.46 \mathrm{MeV}$ (1.7\% relative energy spread at $27 \mathrm{MeV})$. Yet, the most remarkable feature of this
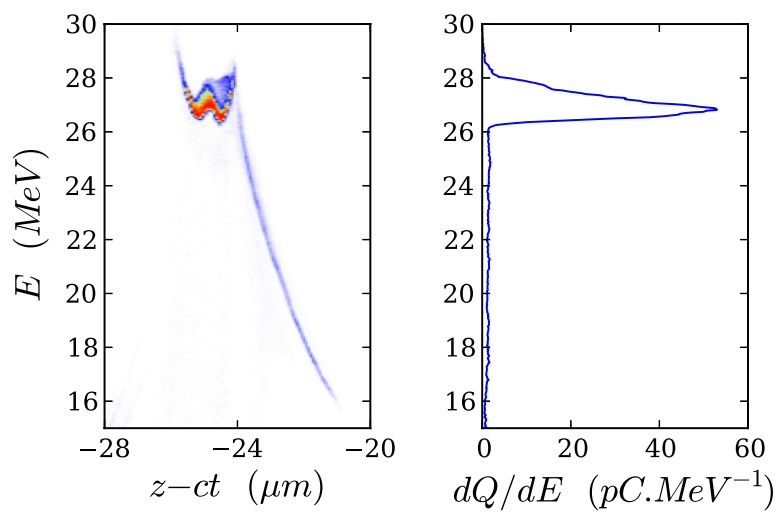

FIG. 2 (color online). Repartition of the injected electrons in the longitudinal (top) and transverse (bottom) phase space, after $0.15 \mathrm{~mm}$ of acceleration. The coordinates on the $x$ axis of the left panel are the same as that of Fig. 1.

injection, and its main advantage over other optical injection methods, is its very low normalized transverse emittance. (See the Supplemental Material [38] for a representation of the transverse phase space of the bunch.) For the electrons in the monoenergetic peak, we obtained $\epsilon_{x} \approx \epsilon_{y} \approx 0.17 \mathrm{~mm} \cdot \mathrm{mrad}$. By comparison, the emittance of the electrons in the tail is $1.9 \mathrm{~mm} \cdot \mathrm{mrad}$. We suggest that the very low emittance obtained here is due to the fact that the electrons are injected at the very back of the bubble. If $\xi_{\text {inj }}$ is the longitudinal position of injection with respect to the back of the bubble, the transverse radius of the bubble at this position $r_{b}\left(\xi_{\text {inj }}\right)$ can be very narrow for small $\xi_{\text {inj }}$ (see Fig. 3). This limits the maximal radius of the betatron motion for the injected electrons (see the Supplemental Material [39] for more details) and thus imposes a cap on the emittance of the electrons. A rough estimate of this cap can be found by assuming that in the 4D transverse phase space, the electrons are uniformly distributed in a 4D ellipse of equation $y^{2}+z^{2}+\left(p_{y}^{2}+\right.$ $\left.p_{z}^{2}\right) / \gamma_{\text {inj }}^{2} k_{\beta}^{2} \leq r_{b}\left(\xi_{\text {inj }}\right)^{2}$, where $k_{\beta}=k_{p} / \sqrt{2 \gamma_{\text {inj }}}$ is the betatron wave vector. This leads to a maximal emittance $\epsilon_{\perp, \max }=\gamma_{\text {inj }} k_{\beta} r_{b}\left(\xi_{\text {inj }}\right)^{2} / 6$. This rough estimate can be used to explain why the emittance of the electrons in the peak $\left[\xi_{\text {inj }} \approx 1 \mu \mathrm{m}, r_{b}\left(\xi_{\text {inj }}\right) \approx 2 \mu \mathrm{m}\right]$ is much lower than that of the electrons in the tail $\left[\xi_{\text {inj }} \approx 4 \mu \mathrm{m}\right.$, $\left.r_{b}\left(\xi_{\text {inj }}\right) \approx 5 \mu \mathrm{m}\right]$.

According to our analysis, the existence of optical transverse injection requires the deformation of the whole bubble. Thus, the main parameters that control transverse injection are the parameters of the colliding pulse $a_{1}$ and $w_{1}$. In particular, $w_{1}$ controls the transition between a regime dominated by transverse injection and a regime of cold injection. This is because, as suggested by Eq. (4), the collision only affects the electrons with $r \lesssim w_{1}$. For $w_{1} \ll$ $w_{0}$ (as is the case in Ref. [27]), the off-axis electrons that will form the sheath of the bubble are not affected. As a result, the bubble does not deform and no transverse 

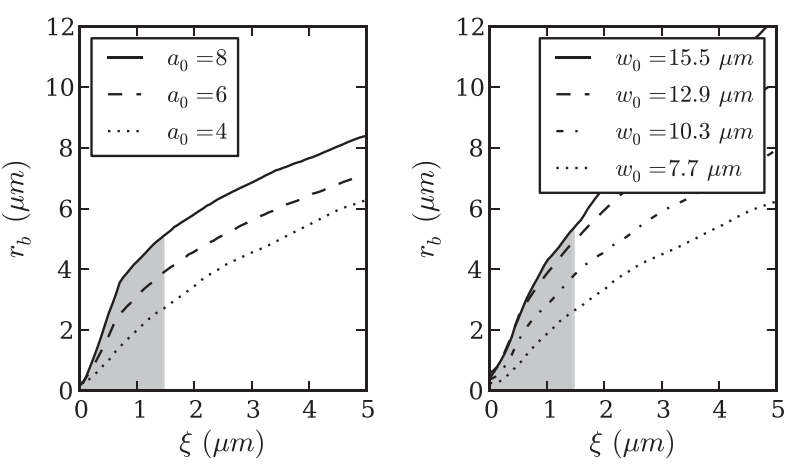

FIG. 3. Profile of the back of the bubble, from simulations for $n=1.75 \times 10^{18} \mathrm{~cm}^{-3}$ and various values of $a_{0}$ and $w_{0}$. The left panel corresponds to $w_{0}=7.8 \mu \mathrm{m}$ and varying values of $a_{0}$, while the right panel corresponds to $a_{0}=4$ and varying values of $w_{0}$. The shaded area represents the typical positions where electrons can be injected through optical transverse injection.

injection occurs. However, the near-axis electrons are affected by the collision and can be injected through cold (longitudinal) injection. On the other hand, for $w_{0} \sim w_{1}$, electrons from all radii are affected by the collision. The bubble strongly deforms and transverse injection dominates. (Longitudinal injection still occurs and is responsible for the tail of the spectrum in Fig. 2.)

Provided that $a_{1}$ and $w_{1}$ are sufficiently large $\left(w_{1} \sim w_{0}\right.$, $\left.a_{1} \gtrsim 0.1\right)$ for transverse injection to occur, the parameters $n, w_{0}$, and $a_{0}$ were observed to mainly control the resulting bunch quality. In fact, as long as the parameters correspond to the bubble regime $\left(k_{p} w_{0} \sim \sqrt{a_{0}}\right.$ [40]), there is a wide range for $n, w_{0}$, and $a_{0}$ in which transverse injection occurs. However, simulations revealed that having $a_{0} \gg$ 4 and $k_{p} w_{0} \gg 2$ produced bunches with relatively high emittance and a large energy spread. We interpret this by the fact that a higher $a_{0}$ or $w_{0}$ leads to a higher value for $r_{b}\left(\xi_{\text {inj }}\right)$ (as shown in Fig. 3) and thus relaxes the cap on the emittance. Conversely, the set of parameters $k_{p} w_{0}=2$ and $a_{0}=4$ leads to high-quality bunches over a wide range of density. (Simulations were run over the range $1.75 \times$ $10^{18}-2 \times 10^{17} \mathrm{~cm}^{-3}$.) Notice that the condition $k_{p} w_{0}=$ 2 entails the use of a tightly focused laser and a low plasma density. This, in turn, implies that the laser will quickly diffract, thereby preventing long acceleration distances and limiting the maximal energy of the electrons to a few tens of $\mathrm{MeV}$ if no external guiding of the laser is used.

We remark that, experimentally, this regime of parameters has not yet been probed. (Previous colliding-pulse experiments used higher density and a wider waist for the driving pulse.) We emphasize, however, that the required pulse energy at $1.75 \times 10^{18} \mathrm{~cm}^{-3}(1.0 \mathrm{~J})$ is available at a number of existing laser facilities. Going towards even lower density tends to produce more charge $(\simeq 100 \mathrm{pC})$ but requires higher laser energy, since satisfying $k_{p} w_{0}=2$ then requires a larger waist. Notice that nowadays there is precisely a global trend towards lower plasma density and higher laser energy, and therefore future experiments will naturally meet the conditions required for high-quality optical transverse injection. Our simulations show that in future colliding-pulse experiments performed in the aforementioned regime of parameters, optical transverse injection will be the prevailing injection mechanism in terms of charge (provided that $w_{1} \geq w_{0}$, which is very likely).

In conclusion, a new paradigm of colliding-pulse injection, which cannot be described by using the 1D separatrix, was presented. In this paradigm, the colliding pulse causes the bubble to shrink and reexpand, which then triggers transverse injection. This injection produces bunches with high charge, short duration, low energy dispersion, and low emittance at the same time. Meeting all these requirements simultaneously is very challenging with other methods of injection. While the energy of the electrons produced may seem low compared to the typical values for laser-wakefield acceleration $(\sim 1 \mathrm{GeV})$, we emphasize that the production of high-quality electron beams at intermediate energies is of interest for a broad range of applications. Such electrons have, for instance, been proposed for the injector of a multistage accelerator [41], as well as for innovative all-optical free-electronlaser schemes [42-44], in which their high quality and relatively low energy are decisive for reaching high gains.

This work was partially supported by the European Research Council through the PARIS ERC Project (Contract No. 226424).

*Corresponding author. remi.lehe@ensta.fr

[1] J. Faure, Y. Glinec, A. Pukhov, S. Kiselev, S. Gordienko, E. Lefebvre, J.-P. Rousseau, F. Burgy, and V. Malka, Nature (London) 431, 541 (2004).

[2] C. G. R. Geddes, C. Toth, J. van Tilborg, E. Esarey, C. B. Schroeder, D. Bruhwiler, C. Nieter, J. Cary, and W.P. Leemans, Nature (London) 431, 538 (2004).

[3] S.P.D. Mangles, C.D. Murphy, Z. Najmudin, A. G. R. Thomas, J. L. Collier, A.E. Dangor, E. J. Divall, P. S. Foster, J. G. Gallacher, C. J. Hooker et al., Nature (London) 431, 535 (2004).

[4] W. P. Leemans, B. Nagler, A. J. Gonsalves, C. Toth, K. Nakamura, C. G. R. Geddes, E. Esarey, C. B. Schroeder, and S. M. Hooker, Nat. Phys. 2, 696 (2006).

[5] V. Malka, J. Faure, Y. A. Gauduel, E. Lefebvre, A. Rousse, and K. T. Phuoc, Nat. Phys. 4, 447 (2008).

[6] K. Nakajima, Nat. Phys. 4, 92 (2008).

[7] M. Xie, Nucl. Instrum. Methods Phys. Res., Sect. A 445, 59 (2000).

[8] S. P. D. Mangles, G. Genoud, M. S. Bloom, M. Burza, Z. Najmudin, A. Persson, K. Svensson, A. G. R. Thomas, and C.-G. Wahlström, Phys. Rev. ST Accel. Beams 15, 011302 (2012).

[9] S. Kneip, C. McGuffey, J.L. Martins, M. S. Bloom, V. Chvykov, F. Dollar, R. Fonseca, S. Jolly, G. Kalintchenko, 
K. Krushelnick et al., Phys. Rev. ST Accel. Beams 15, 021302 (2012).

[10] S. Corde, C. Thaury, A. Lifschitz, G. Lambert, K. Ta Phuoc, X. Davoine, R. Lehe, D. Douillet, A. Rousse, and V. Malka, Nat. Commun. 4, 1501 (2013).

[11] G. R. Plateau, C. G. R. Geddes, D. B. Thorn, M. Chen, C. Benedetti, E. Esarey, A. J. Gonsalves, N.H. Matlis, K. Nakamura, C. B. Schroeder et al., Phys. Rev. Lett. 109, 064802 (2012).

[12] A. J. Gonsalves, K. Nakamura, C. Lin, D. Panasenko, S. Shiraishi, T. Sokollik, C. Benedetti, C. B. Schroeder, C. G. R. Geddes, J. van Tilborg et al., Nat. Phys. 7, 862 (2011).

[13] H. Suk, N. Barov, J. B. Rosenzweig, and E. Esarey, Phys. Rev. Lett. 86, 1011 (2001).

[14] B. B. Pollock, C. E. Clayton, J.E. Ralph, F. Albert, A. Davidson, L. Divol, C. Filip, S. H. Glenzer, K. Herpoldt, W. Lu et al., Phys. Rev. Lett. 107, 045001 (2011).

[15] B. Hidding, G. Pretzler, J. B. Rosenzweig, T. Königstein, D. Schiller, and D. L. Bruhwiler, Phys. Rev. Lett. 108, 035001 (2012).

[16] B. Hidding, J. B. Rosenzweig, Y. Xi, B. O'Shea, G. Andonian, D. Schiller, S. Barber, O. Williams, G. Pretzler, T. Konigstein et al., AIP Conf. Proc. 1507, 570 (2012).

[17] D. Umstadter, J. K. Kim, and E. Dodd, Phys. Rev. Lett. 76, 2073 (1996).

[18] E. Esarey, R. F. Hubbard, W. P. Leemans, A. Ting, and P. Sprangle, Phys. Rev. Lett. 79, 2682 (1997).

[19] R. G. Hemker, K.-C. Tzeng, W. B. Mori, C. E. Clayton, and T. Katsouleas, Phys. Rev. E 57, 5920 (1998).

[20] J. Faure, C. Rechatin, A. Norlin, A. Lifschitz, Y. Glinec, and V. Malka, Nature (London) 444, 737 (2006).

[21] S. Corde, K. T. Phuoc, R. Fitour, J. Faure, A. Tafzi, J. P. Goddet, V. Malka, and A. Rousse, Phys. Rev. Lett. 107, 255003 (2011).

[22] G. Fubiani, E. Esarey, C. B. Schroeder, and W. P. Leemans, Phys. Rev. E 70, 016402 (2004).

[23] H. Kotaki, S. Masuda, M. Kando, J. K. Koga, and K. Nakajima, Phys. Plasmas 11, 3296 (2004).

[24] X. Davoine, E. Lefebvre, J. Faure, C. Rechatin, and A. Lifschitz, Phys. Plasmas 15, 113102 (2008).

[25] X. Davoine, E. Lefebvre, C. Rechatin, J. Faure, and V. Malka, Phys. Rev. Lett. 102, 065001 (2009).
[26] X. Davoine, A. Beck, A. Lifschitz, V. Malka, and E. Lefebvre, New J. Phys. 12, 095010 (2010).

[27] A. Beck, X. Davoine, and E. Lefebvre, New J. Phys. 13, 093016 (2011).

[28] C. Rechatin, J. Faure, A. Lifschitz, V. Malka, and E. Lefebvre, Phys. Plasmas 14, 060702 (2007).

[29] S. Kalmykov, S. A. Yi, V. Khudik, and G. Shvets, Phys. Rev. Lett. 103, 135004 (2009).

[30] S. A. Yi, V. Khudik, S. Y. Kalmykov, and G. Shvets, Plasma Phys. Controlled Fusion 53, 014012 (2011).

[31] I. Kostyukov, E. Nerush, A. Pukhov, and V. Seredov, New J. Phys. 12, 045009 (2010).

[32] S. A. Yi, V. Khudik, C. Siemon, and G. Shvets, Phys. Plasmas 20, 013108 (2013).

[33] J. Vieira, S. F. Martins, V. B. Pathak, R. A. Fonseca, W. B. Mori, and L. O. Silva, Phys. Rev. Lett. 106, 225001 (2011).

[34] J. Vieira, J. L. Martins, V. B. Pathak, R. A. Fonseca, W. B. Mori, and L. O. Silva, Plasma Phys. Controlled Fusion 54, 124044 (2012).

[35] A. F. Lifschitz, X. Davoine, E. Lefebvre, J. Faure, C. Rechatin, and V. Malka, J. Comput. Phys. 228, 1803 (2009).

[36] R. Lehe, A. Lifschitz, C. Thaury, V. Malka, and X. Davoine, Phys. Rev. ST Accel. Beams 16, 021301 (2013).

[37] P. Mora, J. Thomas, and M. Antonsen, Phys. Plasmas 4, 217 (1997).

[38] See Supplemental Material at http://link.aps.org/ supplemental/10.1103/PhysRevLett.111.085005 for a representation of the transverse phase space of the bunch.

[39] See Supplemental Material at http://link.aps.org/ supplemental/10.1103/PhysRevLett.111.085005 for a dynamical representation of the injection process.

[40] W. Lu, C. Huang, M. Zhou, W. B. Mori, and T. Katsouleas, Phys. Rev. Lett. 96, 165002 (2006).

[41] V. Malka, A. Lifschitz, J. Faure, and Y. Glinec, Phys. Rev. ST Accel. Beams 9, 091301 (2006).

[42] A. Bacci, C. Maroli, V. Petrillo, A. Rossi, L. Serafini, and P. Tomassini, Nucl. Instrum. Methods Phys. Res., Sect. A 587, 388 (2008).

[43] V. Petrillo, L. Serafini, and P. Tomassini, Phys. Rev. ST Accel. Beams 11, 070703 (2008).

[44] I. A. Andriyash, E. d'Humières, V. T. Tikhonchuk, and P. Balcou, Phys. Rev. Lett. 109, 244802 (2012). 